\title{
Biological Evaluation of Dilactone Lignan Analogues of Phellinsin A as Chitin Synthase II Inhibitors
}

\author{
Sangku Lee, ${ }^{-}$Jae Nyoung Kim, ${ }^{\dagger}$ Eungsoo Kim, Min-Seok Kim, and Hyeong Kyu Lee ${ }^{-}$ \\ Natural Medicine Research Center, KRIBB. Daejeon 305-806, Korea \\ ${ }^{*}$ E-mail: sangkuathibb.re.kr (SL); hikveeakribb.re.kr (HKL) \\ ${ }^{\dagger}$ Department of Chemistry and Institute of Basic Science. Chonnam Vational Lniversin. Guangiu 500-757, Korea \\ Received September 21, 2009, Accepted October 19, 2009
}

Key Words: Structure-activity relationship, Inhibition. Chitin, Lignan

Chitin is a structural polymer that constitutes the cell walls of nearly all fungi. and play's a crucial role in the determination of cell morphology. There are three different chitin synthases in Saccharomuces cerevisiae. "Chitin synthase I is nonessential repair enzy'me of damaged chitin. ${ }^{3}$ Chitin synthase II is an essential enzyme for the formation of primary septum formation and cell division ${ }^{-}$and chitin synthase III makes more than $90 \%$ of cellular chitin including the chitin synthesized during mating and spoluration." Accordingly. specific inhibitors of chitin synthase II are expected to be an attractive target for the development of antifungal agents.

In the course of our search for a potent antifingal agent from microbial sources, phellinsin A (1) was isolated from the cultured broth of Phellintss sp. PL3." The compound exhibited inhibition of chitin synthase II with an IC $\mathrm{S}_{5 \mathrm{i}}$ value of $28 \mu \mathrm{g} / \mathrm{mL}$ and showed 2.5 times stronger inhibitory activity than polyoxin D The structure of phellinsin A was elucidated by NMR analy'sis and its total synthesis. ${ }^{6}$ Structure-activity relationships for phellinsin A by changing the number of phenolic $\mathrm{OH}$ groups showed that compounds possessing three free phenolic $\mathrm{OH}$ groups in each aryl group of phellinsin A exhibited more potent activity than those of one $\mathrm{OH}$ group or two $\mathrm{OH}$ groups. Our efforts toward the development of a potent antifungal agent have attention to dilactone lignan analogues of phellinsin $\mathrm{A}$. Herein, we describe the biological evaluation of dilactone lignan analogues of phellinsin $\mathrm{A}$.

Dilactone lignan analogues 2 of phellinsin A were prepared by oxidative dimerization of the corresponding cinnamic acid derivatives using either $\mathrm{FeCl}_{3}^{8}$ or $\mathrm{PbO}_{2}{ }^{8}$ as an oxidant. Thermodynamically more stable cis-ring fused-dilactones positioning exo-orientation of the aryl groups were formed. The dilactones (2a, 2c, 2d. 2e) involving para-hydroxy-substituted aryll moieties except $\mathbf{2 b}$ were prepared by $\mathrm{FeCl}_{3}$, whereas nonphenolic dilactones ( $2 \mathbf{f}$ and $\mathbf{2 g}$ ) and compound $\mathbf{2 b}$ were prepared using $\mathrm{PbO}_{.}{ }^{6}$ Dilactones $\mathbf{2} \mathbf{h}$ and $\mathbf{2} \mathbf{i}$ were prepared from $\mathbf{2 d}$ and 2e by treatment with diazomethane. respectively:

Mono- di-. or tri-phenolic OH substituted dilactones $\mathbf{2 a - e}$ and non-phenolic analogues $2 \mathbf{f}-\mathbf{i}$ were examined in order to investigate the effect of the number of free phenolic $\mathrm{OH}$ groups in dilactone analogues 2 of phellinsin A on chitin sy nthase II inhibitory activity. Inhibitory activities of chitin synthase II for the compounds were evaluated by measurement of the formation of chitin with UDP-[ $\left.{ }^{14} \mathrm{C}\right]-\mathrm{N}$-acetyl-D-glucosamine.
Table 1 showed the inlubitory activities of dilactone analogues at 140 and $280 \mu \mathrm{g} / \mathrm{mL}$ concentrations. The activities were largely dependent on the presence of free phenolic $\mathrm{OH}$ substituents in dilactone analogues 2 . Generally the compounds with nonphenolic $\mathrm{OH}$ groups (2f, 2g. 2h. 2i) did not show inhibitory activities at $140 \mu \mathrm{g} / \mathrm{mL}$ concentration. Compounds with two and three phenolic $\mathrm{OH}$ groups ( $\mathbf{2} \mathbf{a}$ and $\mathbf{2 b}$ ) exhibited potent activities. whereas compounds with one phenolic $\mathrm{OH}$ group (2c) did not show inhibitory activities at $140 \mu \mathrm{g} / \mathrm{mL}$ concentration. Interestingly. dilactone $2 \mathrm{~d}$ possessing one phenolic $\mathrm{OH}$ group and an additional methosy group at the each aryl moiety showed good inhibitory activity $(68.9 \%$ ) at $140 \mu \mathrm{g} / \mathrm{mL}$ concentration. However, dilactone $2 \mathrm{e}$ with one phenolic $\mathrm{OH}$ group and two additional methoxy groups did not show activities at $140 \mu \mathrm{g} / \mathrm{nL}$ concentration. Inhibitory activities of compounds exhibiting potent activities at $140 \mu \mathrm{g} / \mathrm{mL}$ concentration were confirmed by the positive control with polyoxin $\mathrm{D}$ (Table 2). As

Table 1. Inhibitory activities of chitin synthase II for dilactone lignan 2

\begin{tabular}{cccccc}
\hline $\mathrm{R}_{1}$ & $\mathrm{R}_{2}$ & $\mathrm{R}_{3}$ & $\mathbf{2}$ & \% Inhibition & \% \\
\hline $\mathrm{H}$ & $\mathrm{OH}$ & $\mathrm{OH}$ & $\mathbf{2 a}$ & 89.7 & - \\
$\mathrm{OH}$ & $\mathrm{OH}$ & $\mathrm{OH}$ & $\mathbf{2 b}$ & 79.9 & - \\
$\mathrm{H}$ & $\mathrm{OH}$ & $\mathrm{H}$ & $\mathbf{2 c}$ & $\mathrm{NA}$ & 18.6 \\
$\mathrm{H}$ & $\mathrm{OH}$ & $\mathrm{OCH}_{3}$ & $\mathbf{2 d}$ & 68.9 & - \\
$\mathrm{OCH}_{3}$ & $\mathrm{OH}$ & $\mathrm{OCH}_{3}$ & $\mathbf{2 e}$ & $\mathrm{NA}$ & 6.8 \\
$\mathrm{H}$ & $\mathrm{H}$ & $\mathrm{H}$ & $\mathbf{2 f}$ & $\mathrm{NA}$ & 9.6 \\
$\mathrm{H}$ & $\mathrm{OC}_{2} \mathrm{H}_{4}$ & $\mathrm{H}$ & $\mathbf{2 g}$ & $\mathrm{NA}$ & 14.1 \\
$\mathrm{H}$ & $\mathrm{OCH}_{3}$ & $\mathrm{OCH}_{3}$ & $\mathbf{2 h}$ & $\mathrm{NA}$ & $\mathbf{1 0 . 1}$ \\
$\mathrm{OCH}_{3}$ & $\mathrm{OCH}_{3}$ & $\mathrm{OCH}_{3}$ & $\mathbf{2 i}$ & NA & 7.9 \\
\hline
\end{tabular}

${ }^{a}$ Percent inhibition at $\mathrm{l} 40 \mu \mathrm{g} \mathrm{mL}$. ${ }^{\text {Percent inhibition at } 280 \mu \mathrm{g} \mathrm{mL} .{ }^{\circ} \mathrm{Not}}$ determined: NA, no inhibitory activity at $140 \mathrm{~kg} \mathrm{~mL}$.

Table 2. IC ${ }_{\xi||}$ values of selected compounds

\begin{tabular}{cc}
\hline Compound & IC $_{50}(\mu \mathrm{g} / \mathrm{mL})$ \\
\hline $2 \mathbf{a}$ & 8.2 \\
$2 \mathbf{b}$ & 40.8 \\
$\mathbf{P A}^{a}$ & 27.0 \\
$\mathbf{P D}^{2}$ & 70.0 \\
\hline
\end{tabular}

${ }^{a}$ Phellinsin $\mathrm{A}$ as a reference. ${ }^{b} \mathrm{Polyoxin} \mathrm{D}$ as a reference. 


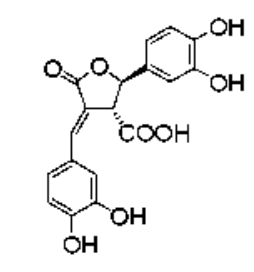

Phellinsin A (1)

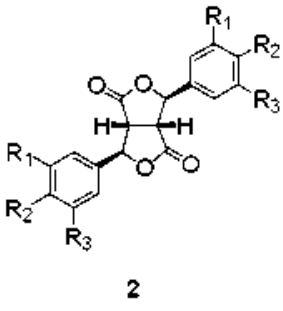

Figure 1. Phellinsin A (1) and dilactone analogues 2.

showed in Table 2. dilactone 2 a possessing two phenolic $\mathrm{OH}$ groups exhibited stronger activity than dilactone $\mathbf{2} \mathbf{b}$ possessing three phenolic $\mathrm{OH}$ groups. Among the tested compounds. dihydroxyphenolic dilactone 2 a showed the most potent activity with an $\mathrm{IC}_{5 \text { (1 }}$ value of $8.2 \mu \mathrm{g} / \mathrm{mL}$. which is about 3 times stronger inhibitory activity than phellinsin A (1). Structure-activity analy'sis indicated that the presence of two free phenolic $\mathrm{OH}$ groups in each aryl group of phellinsin A dilactone analogues contributed to increase the activity. And. the dilactone form 2 a of phellinsin A possessing two phenolic $\mathrm{OH}$ groups increased the activity as compared to the corresponding mono-lactone form. phellinsin A (1)

In conclusion. the effects of the number of free phenolic $\mathrm{OH}$ groups in dilactone lignan analogues of phellinsin $\mathrm{A}$ on inhibitory activities of chitin synthase II were examined for structure-activity relationships. Dilactone analogues lacking free phenolic $\mathrm{OH}$ functionality exhibited poor inhibitory activities. Compounds possessing two free phenolic $\mathrm{OH}$ groups in each aryl group of dilactone $\mathbf{2}$ showed more potent activity than those of one $\mathrm{OH}$ group or three $\mathrm{OH}$ groups. Dihydroxyphenolic dilactone 2 a exhibited about 3 times stronger inhibitory activity than phellinsin $A(1)$ and about 8 times more potent activity than polyoxin $\mathrm{D}$.

\section{Experimental Section}

Compound 2a. To a solution of ferric chloride $(9.2 \mathrm{~g} .56 .7$ mmol) in ethanol ( $100 \mathrm{~mL}$ ) was added a solution of 3.4-dihydroxycinnamic acid ( 4.6 g. $25.5 \mathrm{mmol})$ in ethanol $(30 \mathrm{~mL})$ at room temperature. The reaction mixture was stirred for $\mathrm{l} \mathrm{h}$ and concentrated in vacuo. The residue was diluted with water. extracted with ethyl acetate. washed with brine. dried over $\mathrm{MgSO}_{4}$, and concentrated in vacuo. The resulting residue was chromatographed on silica gel ( $1: 1$ hexane-EtOAc) to afford $5.0 \mathrm{~g}(55 \%)$ of $2 \mathrm{a}$ as a yellow solid: mp $176-178^{\circ} \mathrm{C}:{ }^{\mathrm{l}} \mathrm{H} \mathrm{NMR}$ (300 MHz, acetone- $d_{6}$ ) ò 8.17 (s. $2 \mathrm{H}$ ). 8.06 (s. $2 \mathrm{H}$ ), 6.90 (dd, $J=8.1,1.8 \mathrm{~Hz}, 2 \mathrm{H}), 6.85$ (s. $2 \mathrm{H}), 6.8 \mathrm{l}(\mathrm{dd} J=8.1,1.8 \mathrm{~Hz}, 2 \mathrm{H})$, 5.72 (brs. 2H), 3.99 (brs, 2H); ${ }^{13} \mathrm{C} \mathrm{NMR}$ (75 MHz, acetone-do) ò $176.0,146.8,146.5,131.3,118.4,116.5,113.9,83.1,49.3$ : HRMS (FAB) $m / z 359.0771\left[(\mathrm{M}+\mathrm{H})^{+}\right.$, calcd for $\mathrm{C}_{18} \mathrm{H}_{15} \mathrm{O}_{8}$ $359.0767]$

Compound $2 \mathbf{b}$. To a solution of trifluoroacetic acid $(3 \mathrm{~mL})$ in methylene chloride ( $18 \mathrm{~mL}$ ) was added $3,4,5$-trihydroxycinnamic acid ( $1.0 \mathrm{~g} .5 .2 \mathrm{mmol})$ at $0^{\circ} \mathrm{C}$. Then, $\mathrm{PbO}_{2}$ ( $1.24 \mathrm{~g} .5 .2$ mmol) was added and the reaction mixture was sirred for $1 \mathrm{~h}$. The mixture was poured into ethyl acetate $(100 \mathrm{~mL})$, and the organic layer was washed in succession with water saturated $\mathrm{NaHCO}_{3}$ and brine, dried over $\mathrm{MgSO}_{4}$. and concentrated in vacuo. The resulting residue was purified by flash cluromatography on silica gel $\left(5: 1 \mathrm{CH}_{2} \mathrm{Cl}_{2}-\mathrm{MeOH}\right)$ to afford $0.84 \mathrm{~g}(42 \%)$ of $2 \mathbf{b}$ as a dark brown solid: mp decomp.: ${ }^{\mathrm{H}} \mathrm{H}$ NMR $(300 \mathrm{MHz}$. $\left.\mathrm{CD}_{5} \mathrm{OD}\right) \delta 6.33$ (s. $\left.4 \mathrm{H}\right) .5 .61$ (s. 2H). 3.75 (s, 2H): ${ }^{13} \mathrm{C}$ NMR (75 MHz, CD $\left.{ }_{3} \mathrm{OD}\right) \hat{\delta} 175.9,151.6,137.9,132.1,104.5,83.2$.

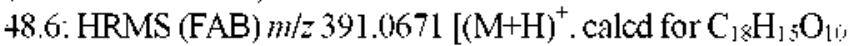
$391.0665]$

Compounds $2 \mathrm{c}-\mathrm{i}$. Compounds $2 \mathrm{c}-\mathrm{i}$ were prepared by the reported method. ${ }^{6}$

2c: A yellow solid: mp $185-188^{\circ} \mathrm{C}:{ }^{\mathrm{l}} \mathrm{H}$ NMR $(300 \mathrm{MHz}$. $\left.\mathrm{CD}_{3} \mathrm{OD}\right) \hat{0} 7.23(\mathrm{dd}, J=6.6 .1 .8 \mathrm{~Hz}, 4 \mathrm{H}) .6 .82(\mathrm{dd}, J=6.6 .1 .8$ $\mathrm{Hz}, 4 \mathrm{H}) .5 .75$ (s. 2H). 3.93 (s. $2 \mathrm{H}):{ }^{15} \mathrm{C}$ NMR $\left(75 \mathrm{MHz}, \mathrm{CD}_{3} \mathrm{OD}\right)$ $\delta 177.3,159.5,130.6 .128 .3 .116 .8 .84 .1 .49 .7$; HRMS (FAB) $m / 2327.085 \mathrm{I}\left[(\mathrm{M}+\mathrm{H})^{+}\right.$. calcd for $\left.\mathrm{C}_{18} \mathrm{H}_{15} \mathrm{O}_{6} 327.0869\right]$.

2d: A white solid: mp $206-208$ "C: ${ }^{1} \mathrm{H}$ NMR $(300 \mathrm{MHz}$, acetone- $\left.d_{6}\right) \delta 7.86(\mathrm{~s}, 2 \mathrm{H}) .7 .06(\mathrm{~d}, J=1.8 \mathrm{~Hz} .2 \mathrm{H}), 6.92$ (dd, $J=7.8 .1 .8 \mathrm{~Hz}, 2 \mathrm{H}$ ). 6.86 (d. $J=7.8 \mathrm{~Hz}, 2 \mathrm{H}$ ). 5.78 (brs. $2 \mathrm{H}$ ). 4.09 (brs, 2H). 3.87 (s. 6H): ${ }^{13} \mathrm{C}$ NMR (75 MHz. acetone- $\left.d_{6}\right) \delta$ 176.1. 148.9, 148.4, 131.0. 119.7, 116.2, 110.5,83.3, 56.5, 49.2: HRMS (FAB) $m / z \quad 387.1092\left[(\mathrm{M}+\mathrm{H})^{-}\right.$, calcd for $\mathrm{C}_{20} \mathrm{H}_{19} \mathrm{O}_{3}$ $387.1080]$.

2e: A pale brown solid: mp $230-234^{\circ} \mathrm{C}:{ }^{1} \mathrm{H}$ NMR $(300 \mathrm{MHz}$. acetone- $d_{6}$ ) $\delta 7.47$ (s. $2 \mathrm{H}$ ), 6.75 (s, $4 \mathrm{H}$ ). 5.76 (brs, 2H), 4.12 (brs. $2 \mathrm{H}) .3 .84$ (s. 12H): ${ }^{13} \mathrm{C}$ NMR $\left(75 \mathrm{MHz}\right.$ acetone- $\left.d_{6}\right)$ oे 176.7 . 149.7. 138.2. 130.5, 105.0.84.1, 57.4.49.8. HRMS (FAB) $m / z$ $447.1288\left[(\mathrm{M}+\mathrm{H})^{-}\right.$, calcd for $\left.\mathrm{C}_{22} \mathrm{H}_{23} \mathrm{O}_{10}, 447.1291\right]$

2f: A pale brown solid: mp $161-163^{\circ} \mathrm{C}:{ }^{1} \mathrm{H} \mathrm{NMR}(300 \mathrm{MHz}$ $\mathrm{CDCl}_{3}$ ) ô $7.44-7.30(\mathrm{nt} .10 \mathrm{H}), 5.93(\mathrm{~s}, 2 \mathrm{H}), 3.56(\mathrm{~s} .2 \mathrm{H}) ;{ }^{13} \mathrm{C}$ NMR $\left(75 \mathrm{MHz}_{2} \mathrm{CDCl}_{3}\right) \delta$ 174.8, 138.0, 129.2. 129.1. 124.5. $81.7,48.2$; HRMS (FAB) $m z 295.0982\left[(\mathrm{M}+\mathrm{H})^{-}\right.$, calcd for $\left.\mathrm{C}_{18} \mathrm{H}_{15} \mathrm{O}_{4} 295.0970\right]$

2g: A white solid; mp $163 \cdot 164{ }^{\circ} \mathrm{C} ;{ }^{1} \mathrm{H}$ NMR $(300 \mathrm{MHz}$, $\left.\mathrm{CDCl}_{3}\right) \hat{o} 7.21(\mathrm{~d}, J=8.7 \mathrm{~Hz} .4 \mathrm{H}) .6 .90(\mathrm{dd}, J=6.6,1.8 \mathrm{~Hz} .4 \mathrm{H})$. 5.88 (s. $2 \mathrm{H}), 4.02(\mathrm{q} . J=6.9 \mathrm{~Hz}, 4 \mathrm{H}), 3.56$ (s. $2 \mathrm{H}) .1 .4 \mathrm{I}(\mathrm{t} . J=$ $6.9 \mathrm{~Hz} .6 \mathrm{H})$ : ${ }^{13} \mathrm{C} \mathrm{NMR}\left(75 \mathrm{MHz} . \mathrm{CDCl}_{3}\right)$ ô $174.9,159.6,129.8$. 126.2, $115.1,81.9,63.7,48.3,14.7$ : HRMS (FAB) $m z 383.1485$ $\left[(\mathrm{M}+\mathrm{H})^{-}\right.$, calcd for $\left.\mathrm{C}_{22} \mathrm{H}_{23} \mathrm{O}_{6} 383.1495\right]$ ]

2h: A white solid: mp $200-203{ }^{\circ} \mathrm{C}$ : ${ }^{1} \mathrm{H}$ NMR $(300 \mathrm{MHz}$. $\left.\mathrm{CDCl}_{3}\right) \delta 7.03-7.00(\mathrm{~m} .6 \mathrm{H}) .5 .80$ (s. $\left.2 \mathrm{H}\right) .4 .22$ (s. $\left.2 \mathrm{H}\right) .3 .79$ (s. $6 \mathrm{H}), 3.77$ (s. $6 \mathrm{H}):{ }^{13} \mathrm{C} \mathrm{NMR}\left(75 \mathrm{MHz}, \mathrm{CDCl}_{3}\right)$ ô 175.3 .149 .4 . $149.0,130.5,118.8,111.7,109.9,81.7,55.7,55.6,48.1$ : HRMS (FAB) $m / z+15.1394\left[(\mathrm{M}+\mathrm{H})^{-}\right.$, calcd for $\left.\mathrm{C}_{2} \mathrm{H}_{23} \mathrm{O}_{8}+15.1393\right]$

2i: A white solid: mp $200-202{ }^{\circ} \mathrm{C}:{ }^{1} \mathrm{H}$ NMR $(300 \mathrm{MHz}$. DMSO- $\left.d_{6}\right)$ o $6.73(\mathrm{~s} .4 \mathrm{H}) .5 .79(\mathrm{~s}, 2 \mathrm{H}), 4.26(\mathrm{~s} .2 \mathrm{H}) .3 .81(\mathrm{~s}$, $12 \mathrm{H}), 3.66(\mathrm{~s} .6 \mathrm{H}):{ }^{12} \mathrm{C}$ NMR $\left(75 \mathrm{MHz}\right.$. DMSO- $\left.d_{6}\right) \delta 175.3$, 153.2. 137.8. 133.9. 103.5.81.6.60.0.56.1, 47.8: HRMS (FAB) $m z+75.1601\left[(\mathrm{M}+\mathrm{H})^{+}\right.$. calcd for $\left.\mathrm{C}_{24} \mathrm{H}_{2}-\mathrm{O}_{10}+75.1604\right]$

Chitin synthase II assay. The strain used in this study is a Saccharomves cerevisiae ECY38-38A ( 14 Ta chsl-23 chs2: LEL 2 callicsd 2 ura3-52 trpl-1 leu2-2 pAS6). which can only overexpress the chitin synthase II. that was grown in YPG (yeast extract $1.0 \%$. peptone $2.0 \%$, galactose $2.0 \%$ ). The cells suspended in $50 \mathrm{mM}$ Tris- $\mathrm{HCl}$ ( $\mathrm{pH} 7.5$ ) containing $5 \mathrm{mM}$ magnesium acetate were broken by vortex mixing with glass beads. ${ }^{10}$ And the cell walls were sedimented at $3.000 \times \mathrm{g}$ for $5 \mathrm{~min}$ and supernatant fluid was centrifuged at $130.000 \times \mathrm{g}$ for $45 \mathrm{nun}$. The membrane pellet was resuspended in the $50 \mathrm{mM}$ Tris- $\mathrm{HCl}$ $(\mathrm{pH} 7.5$ ) containing $33 \%$ glycerol used in the breakage. to a 
final volume of $1.6 \mathrm{~mL} / \mathrm{g}$ (wet weight) of cells used. The activity of chitin synthase II was measured by the described procedure. ${ }^{\text {if }}$ For the proteolytic activation step. reaction mixtures contained $32 \mathrm{mM}$ Tris- $\mathrm{HCl}(\mathrm{pH} 8.0), 1.6 \mathrm{mM}$ cobalt acetate. $1.0 \mathrm{mM}$ UDP- $\left[{ }^{14} \mathrm{C}\right]-G l c N A c(400.000 \mathrm{cpm} / \mathrm{mmol}$. NEN). $2 \mathrm{~mL}$ of typsin at the optimal concentration for activation $(2.0 \mathrm{mg} / \mathrm{mL}), 20$ $\mu \mathrm{L}$ of membrane suspension, and $1+\mu \mathrm{L}$ of samples in a total volume of $46 \mu \mathrm{L}$. The mixtures were preincubated for $15 \mathrm{~min}$ at $30^{\circ} \mathrm{C}$. Proteoly sis was stopped by adding $2 \mu \mathrm{L}$ of a soybean trypsin inhibitor $(4.0 \mathrm{mg} / \mathrm{mL})$ at a concentration 2 times that of trypsin used. and mixtures were placed on ice for $10 \mathrm{~min}$. GlcNAc was added to a final concentration of $32 \mathrm{mM}$ and incubation at $30^{\circ} \mathrm{C}$ was carried out for $90 \mathrm{~min}$. The insoluble chitin formed was assayed by measurement of radioactivity after addition of $10 \%$ trichloroacetic acid and filtration through glass fiber filter (GF/C. Whatman). The concentration of protein was measured by the method of Lowry. ${ }^{11}$ Blank values were measured with addition of $25 \%$ aqueous $\mathrm{MeOH}$ instead of both enzy'me and sample. Percent inhibition of chitin synthase II activity was calculated by substracting the blank values from both control and test sample values using the following equation: Inlibition $(\%)=[1-$ Sample $(\mathrm{cpm})-$ Blank $(\mathrm{cpm}) /$ Control $(\mathrm{cpm})-$ Blank (cpm)] $\times 100$.

Acknowledgments. This research was supported by a grant from KRIBB Research Initiative Program.

\section{Refeiences}

1. Gooday, G. W. J. Gen. Microbiol. 1977,99, 1 .

2. Shaw, J. A.; Mol, P. C.: Bowers, B.; Silverman, S. J.: Valdivieso, M. H.: Duran, A.; Cabib, E. J. Cell Biol 1991, 114, 11 .

3. Cabib, E.: Sburlati, A.; Bowers, B.: Silverman, S. J. J. Cell Biol. $1989,108,1665$.

4. (a) Sburlati, A.: Cabib, E. J. Biol Chem 1986, 261, 15147; (b) Silverman, S. J.: Sburlati, A.: Slater, M. L.; Cábib, E. Proc. Katl. Acad. Sci. L. S. A. 1988, 85, 4735 .

5. Hwang, E-I.: Yun, B.-S.; Kim, Y.-K.; Kwon, B.-M.; Kim, H.-G.: Lee, H.-B.; Jeong, W.-J.; Kim, S.-U. J. Antibiot 2000, $53,903$.

6. Kim, E.; Lee, H. K.; Hwang, E.-I.; Kim, S.-U.; Lee, W. S.; Lee, S.; Jung, S.-H. Symth. Conmmin. 2005, 35, 1231.

7. Lee, S.; Kim, S.-U.; Kim, E.; Hwang, E.-I; Jung, S.-H.; Lee, H. K. Bull. Korean Chent. Soc. 2007, $28,1824$.

8. (a) Cartwright, N. J; Haworth, R. D. J. Chent. Soc. 1944, 535 ; (b) Ahmed, R; Lehrer, M; Stevenson, R. Tetrahedron 1973, 29, 3753; (c) Pelter, A.; Ward, R. S.; Watson, D. J.; Collins, P.; Kay, I. T. Tetrohedron Lett 1979, 2275.

9. Yuzikhin, O. S.; Vasil'ev, A. V; Rudenko, A. P. Russ. J. Org, Chemt. $2000,36,1743$.

10. Choi, W. T.; Cabib, E. Anal Biochent 1994, $219,368$.

11. Lowry; O. H.; Rosebrough, A. L.; Farr, A. L.; Randall, R. J. J. Biol. Chem 1951, 193, 265. 